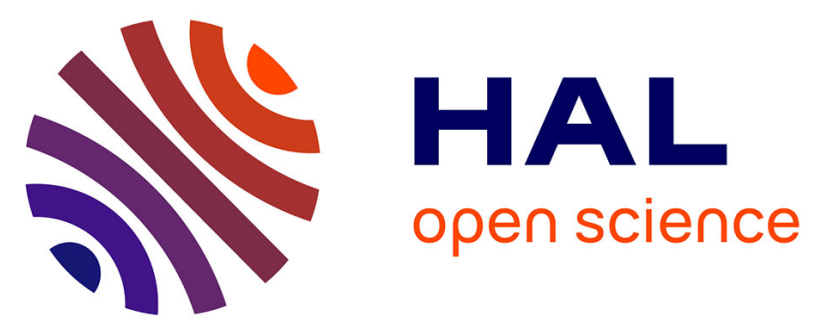

\title{
HIV-1 virological remission lasting more than 12 years after interruption of early antiretroviral therapy in a perinatally infected teenager enrolled in the French ANRS EPF-CO10 paediatric cohort: a case report.
}

Pierre Frange, Albert Faye, Véronique Avettand-Fenoël, Erianna Bellaton, Diane Descamps, Mathieu Angin, Annie David, Sophie Caillat-Zucman, Gilles Peytavin, Catherine Dollfus, et al.

\section{- To cite this version:}

Pierre Frange, Albert Faye, Véronique Avettand-Fenoël, Erianna Bellaton, Diane Descamps, et al.. HIV-1 virological remission lasting more than 12 years after interruption of early antiretroviral therapy in a perinatally infected teenager enrolled in the French ANRS EPF-CO10 paediatric cohort: a case report.. Lancet HIV, 2016, 3 (1), pp.e49-54. 10.1016/S2352-3018(15)00232-5 . pasteur-01289420

\section{HAL Id: pasteur-01289420}

https://hal-pasteur.archives-ouvertes.fr/pasteur-01289420

Submitted on 16 Mar 2016

HAL is a multi-disciplinary open access archive for the deposit and dissemination of scientific research documents, whether they are published or not. The documents may come from teaching and research institutions in France or abroad, or from public or private research centers.
L'archive ouverte pluridisciplinaire HAL, est destinée au dépôt et à la diffusion de documents scientifiques de niveau recherche, publiés ou non, émanant des établissements d'enseignement et de recherche français ou étrangers, des laboratoires publics ou privés.

\section{(2)(1) $\$$}

Distributed under a Creative Commons Attribution - NonCommercial - NoDerivatives 44.0 
HIV-1 virological remission lasting more than 12 years after interruption of early antiretroviral therapy in a perinatally infected teenager enrolled in the French Pediatric Cohort ANRS EPFc010. A case report.

Pierre Frange, $\mathrm{MD}^{1,2,3}$, Prof Albert Faye, $\mathrm{MD}^{4,5}$, Véronique Avettand-Fenoël $\mathrm{PhD}^{2,6}$, Erianna Bellaton, $\mathrm{MD}^{7}$, Prof Diane Descamps, $\mathrm{MD}^{8,9}$, Mathieu Angin, $\mathrm{PhD}^{10}$, Annie David, $\mathrm{PhD}^{10}$, Prof Sophie CaillatZucman, $\mathrm{MD}^{11,12}$, Gilles Peytavin, PharmD ${ }^{9,13}$, Catherine Dollfus, $\mathrm{MD}^{14}$, Jerome Le Chenadec $\mathrm{MSc}^{15}$, Josiane Warszawski, MD ${ }^{15,16}$, Christine Rouzioux, PharmD $D^{2,6}$ and Asier Saez-Cirion, $\mathrm{PhD}^{10}$, on behalf of the ANRS EPF-CO10 Pediatric Cohort and the ANRS EP47 VISCONTI study.

${ }^{1}$ Laboratoire de Microbiologie Clinique, Assistance Publique - Hôpitaux de Paris (AP-HP), Hôpital Necker - Enfants Malades, Paris, France.

${ }^{2}$ EA7327, Université Paris Descartes, Sorbonne Paris Cité, Paris, France.

${ }^{3}$ Unité d'Immunologie, Hématologie et Rhumatologie pédiatriques, AP-HP, Hôpital Necker - Enfants malades, Paris, France.

${ }^{4}$ Service de Pédiatrie générale, AP-HP, Hôpital Robert Debré, Paris, France.

${ }^{5}$ Université Paris Diderot, Sorbonne Paris Cité, Paris, France.

${ }_{7}^{6}$ Laboratoire de Virologie, AP-HP, Hôpital Necker - Enfants Malades, Paris, France.

${ }^{7}$ Service d'Hématologie pédiatrique, AP-HP, Hôpital Robert Debré, Paris, France.

${ }^{8}$ Laboratoire de Virologie, AP-HP, Hôpital Bichat - Claude Bernard, F-75018, Paris, France.

${ }^{9}$ IAME, INSERM UMR1137, Université Paris Diderot, Sorbonne Paris Cité, Paris, France.

${ }^{10}$ Institut Pasteur, Unité HIV Inflammation et Persistance, Paris, France.

${ }^{11}$ Laboratoire d'Immunologie, AP-HP, Hôpital Robert Debré, Paris, France.

12 INSERM UMR1149, Université Paris Diderot, Sorbonne Paris Cité, Paris, France.

${ }_{13}^{13}$ Laboratoire de Pharma-Toxicologie, AP-HP, Hôpital BichaT claude Bernard, F-75018, Paris, France.

${ }^{14}$ Service d'Hématologie et Oncologie pédiatriques, AP-HP, Hôpital Trousseau, Paris, France

${ }^{15}$ INSERM U1018, Université Paris Sud, Le Kremlin Bicêtre, France

${ }^{16}$ Service d'Epidémiologie et de Santé publique, AP-HP, Hôpital Bicêtre, Le Kremlin Bicêtre, France.

Corresponding authors:

Pierre FRANGE, MD, PhD

Laboratoire de Microbiologie Clinique

Hôpital Necker - Enfants malades

149 rue de Sèvres

75015 Paris, France

Telephone: 33.1.44.49.49.61

Fax: 33.1.44.49.49.60

Mail: pierre.frange@aphp.fr

Asier Sáez-Cirión, PhD

Unité HIV Inflammation et Persistance

Institut Pasteur

28 rue du Docteur Roux

75724 Paris Cedex 15, France

Telephone: 33.1.45.68.89.44

Fax: 33.1.45.68.89.57

Mail: asier.saez-cirion@pasteur.fr 
Background: Durable HIV-1 remission after interruption of combined antiretroviral therapy (cART) has been reported in some adults who started cART during primary HIV-1 infection. However the possibility of long-term remission in vertically-infected children remained unclear. Here we report the case of a perinatally-HIV-1-infected adolescent with unprecedented HIV-1 remission.

Methods: The patient was identified among 100 perinatally HIV-infected children followed up in the ANRS EPF CO10 Pediatric Cohort who initiated cART before 6 months of age. HIV-RNA and CD4+ Tcell counts have been monitored since birth. Ultrasensitive HIV-RNA (Biocentric Ultrasensitive Generic HIV-1 RNA Viral Load kit), PBMC-associated HIV-DNA (Biocentric ultrasensitive Generic HIV DNA Cell kit), HIV-specific T-cell responses (production of cytokines and capacity to suppress HIV infection), reactivation of the CD4+ T-cell reservoir (measured by p24 ELISA and HIV RNA in supernatants upon PHA-activation of purified CD4+ T cells), and plasma concentration of antiretrovirals were evaluated after $>11$ years of control off therapy.

Findings: One infant born from a woman with uncontrolled HIV-1 viremia received zidovudine-based prophylaxis during 6 weeks. HIV-RNA and DNA were not detected 3 and 14 days after birth. HIV-DNA was detected at 4 weeks of age. HIV-RNA reached $2.1 \times 10^{6}$ copies $/ \mathrm{ml}$ at 3 months of age and cART was initiated. HIV-RNA was undetectable one month later. CART was discontinued by the family at some time between 5.8 and 6.8 years of age. HIV-RNA was undetectable at 6.8 years of age and cART was not resumed. HIV-RNA has remained $<50$ copies $/ \mathrm{ml}$ and CD4+ T-cell counts stable through 18.3 years of age. After 11 years of control off therapy, HIV-RNA was below 4 copies/ml and HIV-DNA was 2.2 Log copies $/ 10^{6}$ PBMC. HLA genotype showed homozygosity at several loci $\left(A^{\star} 2301\right.$ ;B*1503/4101;C*0210/0802;DRB1*1101-;DQB1*0602-). HIV-specific CD8+ T-cell responses and Tcell activation were weak.

Interpretation: This case suggests that long-term HIV-1 remission is possible in perinatally-infectedearly-treated children, with similar characteristics as reported in adult post-treatment controllers. Further studies will be necessary to understand the mechanisms associated with HIV remission and whether early treatment of infected children may particularly favour the conditions needed to achieve HIV control after treatment discontinuation.

Funding: Study funded by ANRS. 


\section{RESEARCH IN CONTEXT}

\section{Evidence before this study}

We searched PubMed without filters for "HIV", "cure", "remission" and "children". A single report in 2013 described the case of the in utero HIV-1-infected «Mississippi child» who exhibited transient viral control after interrupting very early-initiated CART. After this case was reported several studies pointed to the particular opportunity of tackling HIV infection in children to obtain virological remission. However viremia rebounded in the Mississippi child 27 months later and other recent reports have described viral relapse after treatment interruption in perinatally-infected children. This left unsolved the possibility of long-term remission in HIV-infected children.

\section{Added value of this study}

This study shows that a perinatally-HIV infected young patient who started cART at 3 months of age has been able to control HIV viremia to undetectable levels for over 12 years after treatment was discontinued by the family at around 6.5 years of age. This patient was not able to naturally control viremia without optimal CART early in life as viral load raised to high levels in two episodes of poor treatment compliance. Moreover the patient's MHC background is generally associated with rapid progression in the absence of treatment. HIV DNA is still present in the cells from this patient and low levels of viral reactivation can be achieved in vitro, although at very low levels.

\section{Implications of all the available evidence}

Our case provides the first evidence of durable virological remission in a perinatally-HIV infected adolescent. The clinical history of this patient suggests a decisive role of the duration of cART to achieve a status that then allowed viral remission after treatment discontinuation. Although exceptional, this case of virological remission in a perinatally-HIV infected patient shares some characteristics with HIV adult patients in remission of infection; among them, the early, although not immediate, initiation of antiretroviral treatment. Together with available evidence, this case strongly supports the search for a possible window of opportunity early after HIV infection for CART implementation in children and adults that might lead to the establishment of a viro-immunological context favouring HIV control after treatment discontinuation. 


\section{INTRODUCTION}

HIV-1 replication generally rebounds from latent cellular reservoirs after antiretroviral treatment interruption, but durable remission is sometimes observed when early combined antiretroviral therapy (cART) is discontinued. ${ }^{1}$ Recently, an infant infected by HIV-1 in utero appeared to have been "functionally cured" after rapid initiation of $\mathrm{CART},{ }^{2}$ but viral load rebounded 27 months after cART discontinuation. ${ }^{3}$ We report a case of ongoing virological and immunological control in a perinatally infected teenager who discontinued early cART more than 12 years ago.

Since 1996, 173 HIV-infected children were included at birth in the French Pediatric Cohort. One hundred children initiated cART before 6 months of age. Among them, 15 patients interrupted cART while plasma HIV-RNA was below 500 copies per milliliter and with at least one measurement recorded after treatment interruption. These children received cART during a median duration of 33 months. Thirteen children experienced viral rebound less than 12 months after cART interruption; one child maintained virological control for 3 years; one child showed unprecedented long-term virological remission.

\section{CASE REPORT}

The child was born in 1996 by spontaneous vaginal delivery at 37 weeks +5 days of gestation to an HIV-1-infected woman who had received zalcitabine monotherapy (instead of zidovudine monotherapy because of severe anaemia) from the 13th week of pregnancy. Maternal viral load at delivery was 42 000 copies per milliliter and the CD4+ T cell count was 81 per cubic millimeter. Intrapartum zidovudine prophylaxis was not administered. The newborn received a six-week course of zidovudine, starting on the day of birth. Her plasma HIV RNA load was below 1000 copies per milliliter on day 3 of life (Supplementary Table 1). HIV DNA was undetectable in her peripheral blood mononuclear cells (PBMC) at three days and two weeks of age. PBMC-associated HIV DNA and plasma HIV RNA were detected for the first time at respectively one and two months of age (Figure 1). CART consisting of zidovudine, lamivudine, didanosine and ritonavir was initiated at three months of age, when the plasma HIV RNA load was 2170000 copies per milliliter, driving her viral load to below 500 copies per milliliter one month later. Between 4 and 6.5 months of age her nucleoside reverse transcriptase inhibitors were changed three times because of severe neutropenia. Thereafter, her CART consisted of 
stavudine, didanosine and ritonavir. Her plasma HIV RNA remained undetectable on cART, except for two transient rebounds at 15 and 21 months of age. Each time, interventions to improve treatment adherence re-established virological control, with undetectable viral load two months later. Between 5.8 and 6.8 years of age, the family discontinued her medical follow-up and treatment. Because her viral load remained undetectable at 6.8 years of age, cART was not resumed. At the ages of 11.6 and 13.9 years, low and transient viral blips were observed (510 and 48 copies per milliliter, respectively). The patient has never exhibited symptoms of HIV infection, and her growth and neurological and pubertal development have been normal. Her CD4+ T cell percentage has remained stable. At the time of this report, at age 18.6 years, she has received no antiretroviral drugs for more than 12 years.

Between 2013 and 2015, detailed virological and immunological studies were performed to characterize this unique case of long-term HIV-1 remission in a vertically infected patient (Case A).

\section{METHODS}

\section{Subjects}

Case A was identified among patients followed up in the ANRS CO10 pediatric cohort as the only with long-term HIV remission after treatment interruption.

Case B was a HIV-infected adolescent followed up in the ANRS CO10 pediatric cohort with uncontrolled viremia after treatment discontinuation (CD4 T cell counts of 537 cells $/ \mathrm{mm} 3(20 \%)$ and a viral load of 1,972 HIV RNA copies/ml at the time of the analysis). This patient was treated (ZDV DDI Nelfinavir) since 10 weeks of age for 3.5 years. At the time of the analysis Case B had been off ART for 11 years.

HIC was a treatment naive adult HIV controller from the ANRS CO21 CODEX cohort with a viral load controlled below 400 HIV RNA copies/ml of plasma for more than 5 years at the time of the analysis.

\section{Virological monitoring}

HIV-1 antibody testing was performed by Western blot (Biorad New Lav Blot 1, Bio-Rad, Marnes-laCoquette, France). Recent plasma samples were tested for HIV RNA with the Ultrasensitive Generic HIV-1 RNA Charge Virale kit (Biocentric, Bandol, France) and the Cobas AmpliPrep/COBAS Taqman HIV-1 kit version 2.0 (Roche, Paris, France). ${ }^{4}$ PBMC-associated HIV DNA levels were measured with 
the ultrasensitive Generic HIV DNA Cell kit (Biocentric, Bandol, France). ${ }^{5}$ The virus was subtyped by phylogenetic analysis of the reverse transcriptase gene sequence.

\section{Pharmacological testing}

At 18.3 years of age, to detect non prescribed cART, commercially available antiretrovirals (at least 15 different antiretroviral drugs) were measured in the plasma by liquid chromatography coupled with tandem mass spectrometry (Acquity UPLC/TQD, Waters Corporation Milford, MA, USA) using a modified method. ${ }^{6}$

\section{Immunological studies}

HLA typing was performed by genotype analysis with a standard reverse sequence-specific oligonucleotide method (LABType SSO, One Lambda, Canoga Park, USA).

CD8+ T cell HIV suppressive capacity ex vivo was analyzed as previously described. ${ }^{7,8}$ For intracellular cytokine staining, PBMC were incubated for 6 hours with overlapping peptide pools encompassing HIV-1 clade B gag, pol and nef $(2 \mu \mathrm{g} / \mathrm{mL}$; NIH AIDS Reagent Program). AntiCD28/anti-CD49d costimulation $(1 \mu \mathrm{l} / \mathrm{mL})$ and the anti-CD107a marker were used in each condition. Phorbol myristate acetate (PMA, $80 \mathrm{ng} / \mathrm{mL}$ ) and ionomycin $(1 \mu \mathrm{g} / \mathrm{mL})$ (Sigma-Aldrich, St. Quentin Fallavier, France) were used as a positive control. Golgi stop $(1 \mu \mathrm{g} / \mathrm{mL}$; BD Biosciences, Le Pont de Claix, France) and Brefeldin A (10 $\mu \mathrm{g} / \mathrm{mL}$; Sigma-Aldrich, St. Quentin Fallavier, France) were added 30 minutes after the start of all incubations. Cells were then stained with the LIVE/DEAD Fixable Aqua Dead Cell Stain kit (Life Technologies, Saint Aubin, France) and anti-CD3 and anti-CD8 antibodies. The Cytofix/Cytoperm kit (BD Biosciences, Le Pont de Claix, France) was used for intracellular staining of anti-IFN- $\mathrm{\gamma}$, anti-IL-2 and anti-TNF- $\alpha$.

\section{Detection and amplification of HIV-1 from peripheral blood CD4+ T cells}

One million CD4+ T cells were activated with phytohemagglutinin $(1 \mu \mathrm{g} / \mathrm{mL})$ and IL-2 (100 IU per milliliter). Two days later the cells were washed, mixed with $3 \times 10^{6}$ pooled PHA-activated CD4+ T cells from two healthy donors and cultured for 22 days with IL-2. Viral replication was monitored in the culture supernatant by using the Generic HIV-1 RNA Charge Virale test and by p24 ELISA (ZeptoMetrix Corporation, Buffalo, USA).

\section{Role of the funding source}


The sponsor of the study had no role in study design, data collection, data analysis, data interpretation, or writing of the report. The corresponding author had full access to all the data in the study and had final responsibility for the decision to submit for publication.

\section{RESULTS}

At 3 days of life, Western blot showed reactivity to all (gp160, gp110, p68, p55, gp41, p40, p34, p25) but one (p18) HIV-1 protein. At 17 years of age, Western blot showed strong reactivity to p68, p55, p40, p34 and p24, weaker reactivity to gp160 and gp41, and no reactivity to gp110 or p18. HIV RNA remained undetectable at 16.9, 18.3 and 18.6 years of age (all $<20$ copies per milliliter by the Roche assay and $<9,<4$ and $<7$ copies per milliliter by the Biocentric assay, respectively). PBMC-associated HIV DNA levels were stable at 16.7, 16.9, 18.3 and 18.6 years of age, at between 2.1 and $2.5 \log _{10}$ copies per million PBMC. These values are within the range observed in adult post-treatment controllers of the ANRS VISCONTI study ${ }^{1}$ and among the lowest observed in untreated perinatallyinfected untreated children ${ }^{10}$. The virus belonged to HIV-1 subtype $H$.

At 18.3 years of age, plasma concentrations of all antiretrovirals, measured concomitantly with plasma HIV RNA, were below the respective detection limits, confirming the absence of recent cART use.

The HLA genotype was A*2301/-; B*1503/4101; C*0210/08:02; DRB1*1101/-; DQB1*0602/-. T cell activation marker expression was normal at 18.3 years of age (respectively $15 \%, 21 \%$ and $12 \%$ of $T$ cells were CD3+/HLA-DR+, CD8+/HLA-DR+ and CD4+/HLA-DR+). ${ }^{11}$ CD8+ T cells strongly produced all cytokines tested and degranulated in response to PMA and ionomycin stimulation (Figure 2A, case A). However, responses to pools of HIV-1 peptides were barely detectable (Figure 2A and supplementary figure 1). The HIV-specific response was weaker in the patient than in a viremic perinatally infected adolescent (Case B) and an adult HIV controller (HIC) who were evaluated during the same period $(0.069 \%$ of IFNY + CD8 + T cells from Case A after stimulation with Gag, Pol and Nef pools vs $1.295 \%$ for Case B and $1.079 \%$ for the HIC) (Figure $2 \mathrm{~A}$, and supplementary figure 2). The patient's CD8+ T cells showed no capacity to suppress HIV-1 infection of CD4+ T cells $(0.23$ log p24 decrease CD4 vs CD4:CD8; 0.77 for Case B and 2.68 for the HIC)(Figure 2C) ${ }^{7,8}$ In contrast, her HIVspecific CD4+ $T$ cell response, as estimated in the CD8neg fraction, was within the same range than 
in the Case B control and the HIC $(0.22 \%$ of IFNY+ CD4+ T cells from Case A after stimulation with Gag, Pol and Nef pools vs $0.128 \%$ for Case B and $0.885 \%$ for the HIC)(Figure 2D and supplementary figure 2). Her HIV-specific $\mathrm{T}$ cells did not show superior polyfunctionality (Figures 2B, 2E and supplementary figure 3$)$.

After stimulation of CD4+ T cells, HIV-1 RNA and p24 were detected in the culture supernatant at all time points (Figure 2F). Her CD4+ T cells were strongly susceptible to infection with HIV-1 Bal in vitro (Figure 2C).

\section{DISCUSSION}

We report the case of a perinatally infected teenager who experienced long-term virological remission from HIV-1 infection lasting for more than 12 years after cessation of early cART. As HIV DNA and HIV RNA were undetectable in her blood at age 3 days, the patient was likely infected very late in gestation or during delivery. Of note, we cannot exclude that the AZT-based postnatal prophylaxis could have contributed to the late positivity of HIV-1 PCR in the baby. Indeed, in a recent analysis of the performance of HIV-1 DNA and HIV-1 RNA tests for early diagnosis of perinatal infection in 1567 French infants, $11 \%$ of infected children had negative PCR results at 1 month during antiretroviral prophylaxis $^{12}$. At 3 months of age, her plasma HIV RNA load was very high and her CD4+ T cell percentage severely degraded. This suggested she was infected by a highly replicative and pathogenic virus. Although cART rapidly controlled viremia and normalized the CD4+ T cell percentage, she experienced two viral rebounds, apparently associated with poor adherence, during her second year of life. These episodes showed that the patient was unable to control the virus without treatment at this age and thus suggested that her long-term treatment contributed to creating an optimal situation in which she could achieve durable remission.

The HIV-1 subtype that infected our patient $(\mathrm{H})$ is quite uncommon and almost exclusively found in central Africa. However, this clade did not seem to contribute to the post-treatment control of HIV replication in our patient for two reasons. First, we observed, in our previous reports, similar immunovirological features in children ${ }^{13}$ and adults ${ }^{14}$ infected with strains clustering with subtype $\mathrm{H}$ than in patients harbouring more frequent HIV-1 subtypes. Second, the severe immune deficiency observed in our patient's mother at the time of delivery and the high HIV-1 viremia measured in both 
the mother and the baby (before cART initiation) suggested that the transmitted strain was not less pathogenic than viruses belonging to more frequent subtypes.

Several characteristics of this patient resemble those of adult post-treatment HIV-1 controllers (PTCs) enrolled in the ANRS VISCONTI study ${ }^{1}$ but differ from those of adult HIV controllers. ${ }^{15}$ First, we found homozygosity at several HLA loci, which has been previously linked to more rapid disease progression. ${ }^{16,17}$ Generally unfavourable HLA genotypes are also commonly observed in adult PTCs. ${ }^{1}$ Second, our patient showed weak T cell activation and HIV-specific CD8+ T cell responses. It should be noted that the use of HIV-1 subtype B consensus peptides can underestimate the response in subtype $\mathrm{H}$ infection, but also that the intensity of the patient's HIV-specific CD4+ T cell responses was close to that of the two control patients we analysed. Overall, our results suggest that the CD8+ T cell response is not the major force keeping control of viremia in this patient. The precise mechanisms associated to post-treatment HIV control remain unclear. Recent data obtained in adult patients from the ANRS VISCONTI study suggest that these patients carry NK cells with a particular phenotype and enhanced capacity to inhibit HIV infection. ${ }^{18}$. Whether the innate immune response plays a role in the control of infection in the case of this patient remains to be analysed. Another possibility is that early initiated and prolonged cART favoured the archiving of less fitted or less inducible viruses. The patient still harbors a low frequency of infected cells, from which HIV replication can be induced in vitro, albeit with difficulty. Weak contribution of central memory T cells to the HIV-1 reservoir has been reported in adult PTCs. ${ }^{1}$ Although we did not address this issue in our patient, neonates have much lower frequencies of central-memory-phenotype CD4+ $T$ cells than adults, and this might protect these cell populations from HIV-1 infection during childhood. ${ }^{19,20}$ Moreover, in children who are treated early, transitional memory CD4+ $T$ cells make a larger contribution to the viral reservoir than do longerlasting memory CD4+ $\mathrm{T}$ cells. ${ }^{21}$ However, no cases of long-term post-treatment remission have previously been reported in children, even in those with very low HIV blood cell reservoir levels before cART cessation. ${ }^{22-24}$ As indefinite antiretroviral therapy is recommended for children, only a very small proportion of early-treated, virologically suppressed children therefore undergo treatment interruption, which may explain the reported rarity of childhood PTCs worldwide. Moreover, viral suppression on CART is more difficult to achieve and sustain in children than in adults, ${ }^{25}$ and we cannot therefore exclude the possibility that a lesser proportion of early-treated children fail to achieve PTC status because of poor viral control during treatment. 
The "Mississippi child" maintained undetectable viremia for 27.6 months after cART discontinuation. ${ }^{3}$ Although cART was also initiated very early, there are noteworthy differences with our patient. First, the Mississippi child's plasma HIV RNA load was 19182 copies per milliliter 31 hours after birth, and she may thus have been infected earlier during pregnancy than our patient. Second, our patient was exposed longer to viral antigens after delivery, as the Mississippi child started CART immediately and had detectable viremia for less than a month. This markedly limited the size of the viral reservoir in the Mississippi child, whose HIV DNA levels were lower than in our patient despite much shorter cART. It is unknown whether longer exposure to viral antigens, by experiencing the immune system, might be important for achieving post-treatment remission. Moreover, because the immune system is immature in young children, longer cART might be necessary to achieve an optimal balance between a small pool of infected cells and potent specific immune responses. It is also unknown whether the brief exposure to the virus during the two brief episodes of viral relapse during the second year of life of the patient contributed to the optimal maturation of the immune response against HIV. Finally, it is likely that durable HIV-1 remission is associated with intrinsic host and viral factors that are favoured by early and lengthy cART.

This case shows that early and prolonged cART might be followed by off-therapy viral control lasting several years in some perinatally infected children with no known features associated with natural control. A better understanding of the underlying mechanisms might lead to therapeutic approaches that enable more perinatally infected patients to discontinue treatment and achieve HIV-1 remission for long periods.

\section{CONTRIBUTORS}

All authors made substantial contributions to the conception and design of the work or to the acquisition, analysis, and interpretation of the data:

$\mathrm{PF}, \mathrm{AF}, \mathrm{EB}, \mathrm{CD}, \mathrm{JW}, \mathrm{CR}$ and $\mathrm{AS}-\mathrm{C}$ participated in the conception and design of the study; VA-F, EB, DD, MA, AD, SC-Z, GP, JLC performed experiments and/or collected and analysed data; All authors interpreted data; RPF and AS-C drafted the manuscript including figures and tables; all authors revised it critically for important intellectual content and have approved the final version submitted for 
publication. All authors fulfill ICMJE criteria for authorship, have had access to all the data in the study, and agree to be accountable for the work in ensuring that questions related to its accuracy or integrity are appropriately investigated and resolved.

\section{DECLARATION OF INTERESTS}

PF, VA-F, GP and AS-C have received travel grants, consultancy fees, honoraria, or study grants from various pharmaceutical companies, including Bristol-Myers-Squibb, Gilead Sciences, ViiV Healthcare, Janssen and Merck outside the submitted work. All other authors declare that they have no conflict of interests. 


\section{REFERENCES}

1. Sáez-Cirión A, Bacchus C, Hocqueloux L, et al. Post-treatment HIV-1 controllers with a longterm virological remission after the interruption of early initiated antiretroviral therapy ANRS VISCONTI Study. PLoS Pathog 2013;9:e1003211.

2. Persaud D, Gay G, Ziemniak C, et al. Absence of detectable HIV-1 viremia after treatment cessation in an infant. N Engl J Med 2013;369:1828-35.

3. Luzuriaga K, Gay H, Ziemniak C, et al. Viremic relapse after HIV-1 remission in a perinatally infected child. N Engl J Med 2015;372:786-8.

4. Rouet F, Chaix ML, Nerrienet E, et al. Impact of HIV-1 genetic diversity on plasma HIV-1 RNA Quantification: usefulness of the Agence Nationale de Recherches sur le SIDA secondgeneration long terminal repeat-based real-time reverse transcriptase polymerase chain reaction test. J Acquir Immune Defic Syndr 2007;45:380-8

5. Avettand-Fènoël V, Chaix ML, Blanche $S$, et al. LTR real-time PCR for HIV-1 DNA quantitation in blood cells for early diagnosis in infants born to seropositive mothers treated in HAART area (ANRS CO 01). J Med Virol 2009;81:217-23.

6. Jung BH, Rezk NL, Bridges AS, Corbett AH, Kashuba AD. Simultaneous determination of 17 antiretroviral drugs in human plasma for quantitative analysis with liquid chromatographytandem mass spectrometry. Biomed Chromatogr 2007;21:1095-104.

7. Sáez-Cirión A, Lacabaratz C, Lambotte O, et al. HIV controllers exhibit potent CD8 T cell capacity to suppress HIV infection ex vivo and peculiar cytotoxic T lymphocyte activation phenotype. Proc Natl Acad Sci U S A 2007;104:6776-81.

8. Sáez-Cirión A, Shin SY, Versmisse P, Barré-Sinoussi F, Pancino G. Ex vivo T cell-based HIV supression assay to evaluate HIV-specific CD8+ T cell responses. Nat Proc 2010;5:1033-4.

9. Delamare C, Burgard M, Mayaux MJ, et al. HIV-1 RNA detection in plasma for the diagnosis of infection in neonates. The French Pediatric HIV Infection Study Group. J Acquir Immune Defic Syndr Hum Retrovirol 1997;15:121-5.

10. Scott-Algara, D, Rouzioux, C, Blanche, S, Burgard, M, Didier, C, Rivière, Y, Buseyne, F. In Untreated HIV-1-Infected Children, PBMC-Associated HIV DNA Levels and Cell-Free HIV RNA Levels Are Correlated to Distinct T-lymphocyte Populations. J AIDS 2010; 53:553-563. 
11. Cohen Stuart JW, Hazebergh MD, Hamann D, et al. The dominant source of CD4+ and CD8+ T-cell activation in HIV infection is antigenic stimulation. J Acquir Immune Defic Syndr 2000;25:203-11.

12. Burgard M, Blanche S, Jasseron C, et al. Performance of HIV-1 DNA or HIV-1 RNA tests for early diagnosis of perinatal HIV-1 infection during anti-retroviral prophylaxis. J Pediatr 2012;160:60-6.

13. Delaugerre C, Warszawski J, Chaix ML, et al. Prevalence and risk factors associated with antiretroviral resistance in HIV-1-infected children. J Med Virol 2007;79:1261-9.

14. Frange P, Assoumou L, Descamps D, et al. HIV-1 subtype B-infected MSM may have driven the spread of transmitted resistant strains in France in 2007-12: impact on susceptibility to first-line strategies. J Antimicrob Chemother 2015;70:2084-9.

15. Sáez-Cirión A, Pancino G. HIV controllers: a genetically determined or inducible phenotype? Immunol Rev 2013;254:281-94.

16. Carrington M, Nelson GW, Martin MP, et al. HLA and HIV-1: heterozygote advantage and $\mathrm{B}^{\star} 35-\mathrm{Cw}^{*} 04$ disadvantage. Science 1999;283:1748-52.

17. Tang J, Costello C, Keet IP, et al. HLA class I homozygosity accelerates disease progression in human immunodeficiency virus type 1 infection. AIDS Res Hum Retroviruses 1999;15:31724.

18. Scott-Algara D Didier C, Arnold V, Cummings J-S, Boufassa F, Lambotte O, Hocqueloux L, Sáez-Cirión A, Rouzioux C. Post-Treatment Controllers Have Particular NK Cells With High Anti-HIV Capacity: VISCONTI Study. Conference on Retroviruses and Opportunistic Infections (CROI). February 23-26, 2015 | Seattle, Washington. Abstract 52, session 0-4. Available from: http://www.croiconference.org/sessions/post-treatment-controllers-have-particular-nkcells-high-anti-hiv-capacity-visconti-study.

19. Shearer WT, Rosenblatt HM, Gelman RS, et al. Lymphocyte subsets in healthy children from birth through 18 years of age: the Pediatric AIDS Clinical Trials Group P1009 study. J Allergy Clin Immunol 2003;112:973-80.

20. Schatorjé EJ, Gemen EF, Driessen GJ, Leuvenink J, van Hout RW, de Vries E. Paediatric reference values for the peripheral T cell compartment. Scand J Immunol 2012;75:436-44. 
21. Luzuriaga K, Tabak B, Garber M, et al. HIV type 1 (HIV-1) proviral reservoirs decay continuously under sustained virologic control in HIV-1-infected children who received early treatment. J Infect Dis 2014;210:1529-38.

22. Giacomet V, Trabattoni D, Zanchetta N, et al. No cure of HIV infection in a child despite early treatment and apparent viral clearance. Lancet 2014;384:1320.

23. Martínez-Bonet M, Puertas MC, Fortuny C, et al. Establishment and replenishment of the viral reservoir in perinatally HIV-1-infected children initiating very early antiretroviral therapy. Clin Infect Dis 2015; 61:1169-78

24. Butler KM, Gavin P, Coughlan S, et al. Rapid viral rebound after 4 years of suppressive therapy in a seronegative HIV-1 infected infant treated from birth. Pediatr Infect Dis $\mathrm{J}$ 2015;34:e48-e51.

25. Pursuing Later Treatment Options II (PLATO II) project team for the Collaboration of Observational HIV Epidemiological Research Europe (COHERE), Castro H, Judd A, et al. Risk of triple-class virological failure in children with HIV: a retrospective cohort study. Lancet $2011 ; 377: 1580-7$ 


\section{FIGURE LEGENDS}

\section{Figure 1. Monitoring of HIV-1 viral load and CD4+ T cell counts in the child.}

HIV-1 RNA load (circles) and CD4+ T cell percentages (triangles) during the entire follow-up period, from birth to age 18.6 years. Red circles indicate detectable HIV-1 RNA levels. Blue circles indicate the assay detection limit in case of undetectable viral load. Shaded areas indicate the periods during which the child received antiretroviral drugs. ZDV denotes zidovudine, DDI didanosine, 3TC lamivudine, RTV ritonavir, and D4T stavudine.

\section{Figure 2. HIV-specific $T$ cell responses after $>10$ years of remission}

Panels $A$ and $D$ show the frequencies of CD8+ $T$ cells and CD8- $T$ cells producing interferon gamma (IFNY) in response to PMA/ionomycin stimulation and to pools of gag, pol and nef peptides. The test was performed simultaneously in the patient (Case A, light gray columns), a viremic perinatally infected adolescent (Case B, dark gray columns) and an adult natural HIV controller (HIC, blue columns). Panels $B$ and $E$ show the relative capacity of CD8+ T cells and CD4+ T cells from the same three patients to concomitantly exert (in response to a pool of gag peptides) one, two, three or four functions (IFN- $\gamma$, IL-2 and TNF- $\alpha$ production and degranulation marker CD107a expression). The size of the circles is proportional to the cumulative total response. Panel C shows the levels of HIV-1 p24 detected in culture supernatants after superinfection with HIV-1 BaL of CD4+ T cells from the HIC, the case A patient and the Case B, cultured without or with autologous CD8+ T cells (CD4:CD8 ratio 1:1). The mean and standard deviation of three replicates are shown. Panel F shows levels of autologous HIV-1 RNA (maximum 7810 copies per milliliter, in blue) and p24 (maximum 122 pg per milliliter, in black) in culture supernatants after stimulation of $\mathrm{CD} 4+\mathrm{T}$ cells from Case A patient with phytohemagglutinin. Heterologous feeder CD4+ T cells were added to the culture on day 7. p24 production after activation of CD4+ T cells from Case B patient are shown as a reference (empty triangles, dot lines). 